\title{
Therapy of supraventricular and ventricular arrhythmias in adults with congenital heart disease-narrative review
}

\author{
Kristina Wasmer ${ }^{1}$, Lars Eckardt ${ }^{1}$, Helmut Baumgartner ${ }^{2}$, Julia Köbe $^{1}$ \\ ${ }^{1}$ Department of Cardiology II - Electrophysiology, University Hospital Muenster, Cardiology, Muenster, Germany; ${ }^{2}$ Department of Cardiology III, \\ Division of Adult Congenital and Valvular Heart Disease, University Hospital Muenster, Cardiology, Muenster, Germany \\ Contributions: (I) Conception and design: K Wasmer; (II) Administrative support: None; (III) Provision of study materials or patients: None; (IV) \\ Collection and assembly of data: None; (V) Data analysis and interpretation: None; (VI) Manuscript writing: All authors; (VII) Final approval of \\ manuscript: All authors. \\ Correspondence to: Prof. Dr. med. Kristina Wasmer. Klinik für Kardiologie II: Rhythmologie, Universitätsklinikum Münster (UKM), Albert- \\ Schweitzer-Campus 1, Gebäude A1, 48149 Münster, Germany. Email: wasmerk@ukmuenster.de.
}

\begin{abstract}
Arrhythmias are among the most common late complications in adults with congenital heart disease (ACHD) and a frequent reason for hospital admission. Both, supraventricular and ventricular arrhythmias, not only cause debilitating symptoms, but may be life-threatening by increasing risk of stroke, causing or worsening heart failure and being associated with sudden death. Substrate and risk for arrhythmia differs widely between congenital defects with specific arrhythmias being much more common in some patients than others. Atrial macroreentrant arrhythmias are particularly frequent in patients with atrial septal defects and repair that involves atrial incisions including patients with transposition of the great arteries (TGA) and atrial switch. Accessory pathways and related arrhythmias are often associated with Ebstein's anomaly and congenitally corrected TGA. Monomorphic ventricular arrhythmias occur in patients with ventricular incisions, namely patients with Tetralogy of Fallot. Changes in surgical repair techniques influence arrhythmia prevalence and substrate as well as anatomical access for catheter ablation procedures. In addition, epidemiologic changes associated with improved long-term survival will further increase the prevalence of atrial fibrillation in ACHD. This article summarizes current understanding of prevalence of specific arrhythmias, underlying mechanisms, medical and interventional treatment options and their outcome in ACHD.
\end{abstract}

Keywords: Adult congenital heart disease (ACHD); supraventricular tachycardia; ventricular tachycardia; antiarrhythmic drug therapy; catheter ablation

Submitted Jul 11, 2020. Accepted for publication Jan 19, 2021.

doi: $10.21037 / \mathrm{cdt}-20-634$

View this article at: http://dx.doi.org/10.21037/cdt-20-634

\section{Introduction}

Arrhythmias are among the most common late complications in adults with congenital heart disease (ACHD) and a frequent reason for hospital admission. Both, supraventricular and ventricular arrhythmias, not only cause debilitating symptoms, but may be life-threatening by increasing risk of stroke, causing or worsening heart failure and being associated with sudden death. Supraventricular and ventricular arrhythmias are not equally distributed over the
ACHD population, but show different prevalence in relation to the underlying congenital defect and in association with their specific surgical repair (1). Due to immense improvement in intensive care and operative medicine, pediatric cardiac surgery can now be performed in small infants, rendering palliative shunt surgery prior to definite repair unnecessary in many patients. In addition, less invasive surgical techniques have evolved over time and have led to lower arrhythmia incidence in some defects (2). For example, the Fontan-procedure has been modified several times and 
current surgical techniques are associated with a much lower rate of atrial arrhythmias compared to earlier repair types (3-5). Likewise, arterial switch as opposed to atrial switch in patients with transposition of the great arteries (TGA) has dramatically reduced the incidence of highly symptomatic and sometimes deadly atrial arrhythmias (6,7). Another example of changing arrhythmia substrate is the change in surgical repair technique and earlier repair in patients with Tetralogy of Fallot that has reduced the number of isthmuses that are the basis for monomorphic ventricular tachycardia (VT) in these patients (8). Nevertheless, there remain isthmuses that are anatomically determined like the one between the ventricular septal defect (VSD) and the pulmonary valve that remains important substrate for VT in these patients $(9,10)$. This article summarizes current understanding of prevalence of specific arrhythmias, underlying mechanisms, medical and interventional treatment options and their outcome in ACHD. We present the following article in accordance with the Narrative Review reporting checklist (available at http:// dx.doi.org/10.21037/cdt-20-634).

\section{Supraventricular arrhythmias}

It is estimated that about $50 \%$ of ACHD develop supraventricular tachycardia (SVT) during their lifetime (11). Recognition and timely management of SVT in ACHD are extremely important as they can cause rapid hemodynamic deterioration, heart failure, and sudden cardiac death, particularly in patients with systemic right ventricle or Fontan circulation (12).

The most common SVT in ACHD is intra-atrial reentrant tachycardia, often also referred to as atrial flutter. Atrial fibrillation is increasingly recognized in ACHD as the population is growing and ageing. Patients with congenital heart disease can also develop paroxysmal SVT due to atrioventricular nodal reentrant tachycardia (AVNRT) or atrioventricular tachycardia (AVRT) via an accessory pathway and focal atrial tachycardia (13). Accessory pathways and associated arrhythmias are most often seen in Ebstein's anomaly and congenitally corrected transposition of the great arteries (14-18). Twin AV-nodes are present most often in patients with congenitally corrected TGA $(1,19,20)$ and can give rise to AVNRT in these patients.

\section{Macroreentrant atrial arrbythmias (intra-atrial reentrant tachycardia, atrial flutter)}

Intra-atrial reentrant tachycardia (IART) due to a macro- reentry in the right atrium is the most common arrhythmia mechanism in ACHD. One or more fixed macro-reentrant circuits related to surgical scars in the right atrium and anatomical obstacles constitute the substrate of this arrhythmia in a wide range of CHD. It is particularly common in patients with moderate and complex CHD, being the underlying mechanism in $86 \%$ of patients in a multicenter study (21).

Patients with atrial septal defect (ASD) and Tetralogy of Fallot have atrial incisions from corrective surgery. In addition, a septal suture or patch in ASD patients, intraatrial tunnels in addition to atrial incisions in patients with atrial switch for transposition of the great arteries and in patients with earlier Fontan-surgical palliation provide the substrate for organized macro-reentrant arrhythmias. In addition to defined sutures and patch material, patients with CHD frequently develop fibrosis due to ischemia and stretch as consequence of volume and pressure overload. Incisions, patch material and fibrosis all lead to conduction slowing, the prerequisite for macro-reentrant arrhythmias. Well-recognized macro-reentrant atrial arrhythmias are isthmus-dependent right atrial flutter around the tricuspid valve and incisional tachycardias rotating around an atrial incision or a septal patch. Combinations of these circuits occur in a substantial number of patients. Among other factors, incidence of intra-atrial macro-reentrant tachycardias depend on timing of the corrective surgery and hemodynamic results (22). For example, patients with ASD who underwent corrective surgery in adulthood or no surgery at all, develop IART and atrial fibrillation with an incidence of $50 \%$ (23). If closure occurred prior to age 15 , incidence is only $16 \%$ (24). In patients with TGA and atrial switch procedure, IART occurs with an incidence of $28-37 \%$, while incidence is only $5 \%$ in patients with arterial switch surgery, which is the standard procedure since the early 1990s (7). Likewise, the incidence of atrial arrhythmias has decreased from $100 \% \geq 26$ years after atrio-pulmonary Fontan procedure to $13 \%$ after 10 years with a lateral tunnel to $0 \%$ after 10 years with extracardiac palliation in patients with single ventricle (3).

\section{Atrial fibrillation (AF)}

$\mathrm{AF}$ is the second most common arrhythmia after intraatrial reentrant tachycardia. It is seen more often in simple and moderately complex congenital heart disease as well as in older patients (21). It is the most common arrhythmia in ACHD beyond the age of 50 years. As life expectancy 
increases in the population with $\mathrm{CHD}$, an upsurge in the prevalence of $\mathrm{AF}$ is expected. Data regarding the interplay between IART and AF are inconclusive (25-27). It appears that there is no clear relation between both arrhythmias, they co-exist in about one third of ACHD patients, particularly complex CHD. But ACHD with AF after a documented episode of regular AT appear to develop AF at a younger age compared to patients with only AF. ACHD are assumed to be vulnerable to AF not only because of previously described hemodynamic consequences and scarring but also due to a higher prevalence of cardiovascular risk factors, namely hypertension, obesity, diabetes, and dyslipidemia (28). A distinguishing feature of AF in ACHD is the suspected presence of AF that is of right atrial origin in contrast to typical adult onset $\mathrm{AF}$ that is of left atrial origin (29).

\section{Paroxysmal supraventricular tachycardia: atrioventricular nodal reentrant tachycardias and accessory pathways}

Paroxysmal supraventricular tachycardia due to AVNRT and AVRT are less common arrhythmia mechanisms in the general ACHD population. While the prevalence of AVNRT is less well studied (30), the presence of accessory pathways, and often multiple pathways, is well recognized. It is most often seen in patients with Ebstein's anomaly, but also congenitally corrected transposition of the great arteries (ccTGA) with and without associated malformations. The high prevalence of accessory pathways in these two congenital defects is thought to be a consequence of disturbation in embryological development of the atrioventricular (AV) valves (31). In both defects, accessory pathways are typically located at the inferior aspect of the tricuspid valve which is located on the right side in Ebstein's anomaly and on the left in patients with $\mathrm{AV}$ discordance (i.e., ccTGA). In a retrospective study of patients with Ebstein's anomaly, 91\% of pathways were manifest (referring to antegrade conduction showing a delta-wave on ECG), $35 \%$ of patients had two accessory pathways and the majority was conducting rapidly to the ventricle putting the patients at increased risk for rapidly conducted $\mathrm{AF}$ that can degenerate to ventricular fibrillation leading to sudden cardiac death (15). Accessory pathways of the Mahaim type have also been observed in patients with Ebstein's anomaly. Takeuchi and colleagues (17) recently reported their experience in patients with $\mathrm{AV}$ discordance. Thirteen accessory pathways were present in 11 patients, all of which were located at the anatomical left-sided tricuspid annulus, mainly posteriorly.

\section{Ventricular arrhythmias}

Ventricular arrhythmias can be monomorphic or polymorphic and each of these ECG defined arrhythmias is related to different substrates. Monomorphic VT develop in patients with a defined substrate, namely boundaries formed by dense scar due to patch material, sutures and anatomical obstacles like valves in combination with slowly conducting tissue being again the prerequisite for macro-reentrant arrhythmias. The best investigated congenital defect in this context is Tetralogy of Fallot where certain isthmuses have been related to monomorphic VT $(9,10,32)$. Some of these isthmuses are also present in patients with ventricular septal defect (VSD) and patients with complex TGA, i.e., patients with TGA and VSD (2). These isthmuses are amenable to catheter ablation and measurements of conduction properties during EP-study have been suggested to identify patients at risk (10,32). Polymorphic VT and ventricular fibrillation (VF), on the other hand, occur due to diffuse fibrosis and myocardial disarray. Patients with single ventricle, left ventricular outflow tract obstructive lesions, and patients with failing systemic ventricle are at highest risk $(1,2)$.

\section{Significance of supraventricular and ventricular arrhythmias}

Supraventricular and ventricular arrhythmias are associated not only with debilitating symptoms, but substantial cardiovascular morbidity and mortality (33-37). Even patients with lower complexity CHD are at risk for hemodynamic deterioration due to fast heart rates, development or worsening of systemic ventricular dysfunction and heart failure as well as stroke. Sudden cardiac death is the most devastating event and occurs both due to ventricular arrhythmias and sometimes supraventricular arrhythmias. In a retrospective study on patients with TGA after atrial switch who had received an implantable cardioverter defibrillator (ICD), $50 \%$ of patients had SVT prior to VT (12). $80 \%$ of sudden cardiac deaths in patients with TGA and systemic right ventricle occur during exercise as opposed to only $8-10 \%$ in the general ACHD population (38). It is thought that ischemia of the systemic right ventricular myocardium plays a critical role in pathophysiology of SCD in these patients $(38,39)$.

Prevention and treatment of arrhythmias is of upmost importance in ACHD patient care to decrease morbidity 
and mortality of these patients due to heart failure, stroke and sudden death. Prevention of arrhythmias includes early detection and treatment of CHD, implementation of early and less invasive surgical therapies, and management of cardiovascular risk factors (40). Prevention of complications includes stroke prevention in atrial arrhythmias, rate control, arrhythmia suppression by antiarrhythmic drugs and ideally curative therapy by catheter ablation. Prevention of sudden cardiac death includes monitoring and treatment of hemodynamic problems and systemic ventricular function as well as risk stratification for individual patients. A separate chapter in this issue deals with prevention of sudden cardiac death and ICD therapy in ACHD.

\section{Antiarrhythmic drug therapy}

Despite their well recognized side effects and limited efficacy, antiarrhythmic drugs are an important therapeutic option to prevent or treat arrhythmias in ACHD. There are only few data on antiarrhythmic drug therapy in ACHD patients and guideline recommendations are based on expert opinion and adopted from experiences in patients without congenital heart disease. Use of antiarrhythmic drugs in ACHD is limited by underlying structural heart disease, particularly ventricular dysfunction, but also coexisting brady-arrhythmias. Additional factors like concomitant hepatic and renal dysfunction can alter pharmacokinetics of antiarrhythmic drugs (41). Before terminating tachycardia, 12-lead ECG documentation should be achieved whenever possible. It is the most important diagnostic tool to guide acute management, evaluation of long-term treatment and prevention of recurrences (Figure 1).

\section{Acute management}

Guidelines on arrhythmia management in congenital heart disease $(32,42)$ and on use of antiarrhythmic drugs (43) have recently been published. Acute cardioversion is indicated in unstable patients. In patients without hemodynamic compromise, decision regarding treatment options has to take into account the underlying arrhythmia mechanism, underlying heart defect, prevalence of arrhythmia, patient choice as well as availability of interventional electrophysiologists with expertise in catheter ablation in CHD.

Regular SVT that is thought to be dependent on AVnodal conduction, i.e., AVNRT or AVRT, can acutely be terminated with adenosine. According to current guidelines, use of adenosine, though, is not recommended in patients with low ejection fraction who depend on a high heart rate to preserve cardiac function (32). The duration of $\mathrm{AV}$ block after adenosine administration, though, is only in the range of seconds. In our opinion, it should therefore not pose a problem based on its very brief slowing of heart rate. Anesthesia and electrical cardioversion can be avoided by adenosine administration. Nevertheless, adenosine should be given with a defibrillator at hand in all cases as it can provoke atrial fibrillation which could be rapidly conducted in patients with underlying manifest accessory pathways (43).

In patients with $\mathrm{AF}$ that is conducted via an accessory pathway (hallmark irregular, typically rapidly conducted rhythm with variable QRS width and morphology, so called FBI tachycardia, Fast Broad Irregular, Figure 1C), ajmaline can be administered to terminate $\mathrm{AF}$ and to block the accessory pathway. Ajmaline is a class Ia drug that is used to terminate and prevent wide complex tachycardias, but not included in guidelines for ACHD (1). We recommend electrical cardioversion in ACHD with $\mathrm{AF}$ and conduction via an accessory pathway as the safest option.

Beta-blockers can be used to facilitate spontaneous termination of regular SVT. As they have a negative inotropic effect and can worsen underlying sinus node dysfunction, they should be used with caution in patients with ventricular dysfunction. The same is true for verapamil, a nondihydropyridine calcium channel blocker that slows conduction through the AV-node but also has negative inotropic effects. Choice of antiarrhythmic drug or termination with electrical cardioversion depends on available information regarding CHD and systemic ventricular function as well as hospital setting and individual experience of the responsible physician Despite need for sedation, electrical cardioversion may be the safest option in more complex CHD and the only option in patients who are hemodynamically unstable.

Macro-reentrant atrial arrhythmias are amenable to catheter ablation and are best investigated while ongoing to determine the exact mechanism (see section on catheter ablation). Hemodynamically unstable patients have to be cardioverted, if possible after ECG documentation. In patients who are hemodynamically stable, beta-blocker therapy is first choice to slow heart rate because of its rapid onset of action and effectiveness at high sympathetic tone. Digoxin can be used as well, if sufficient rate control cannot be achieved by beta-blockers or nondihydropyridine calcium channel blockers alone. The 2014 guidelines discuss controversy regarding possible mortality increase with digoxin (1). The most recent ESC guidelines on 

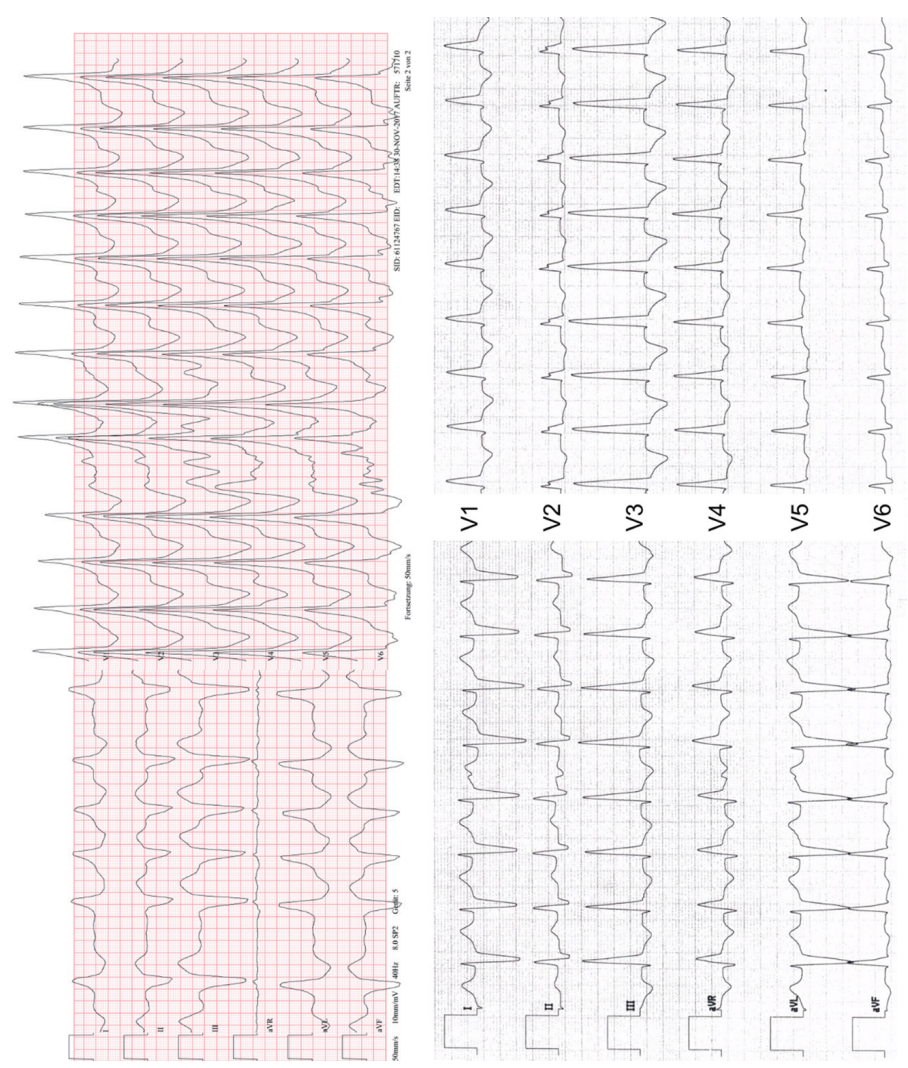

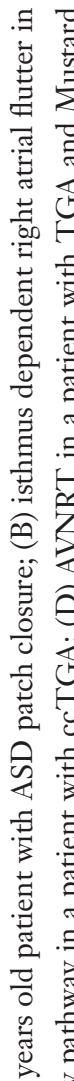
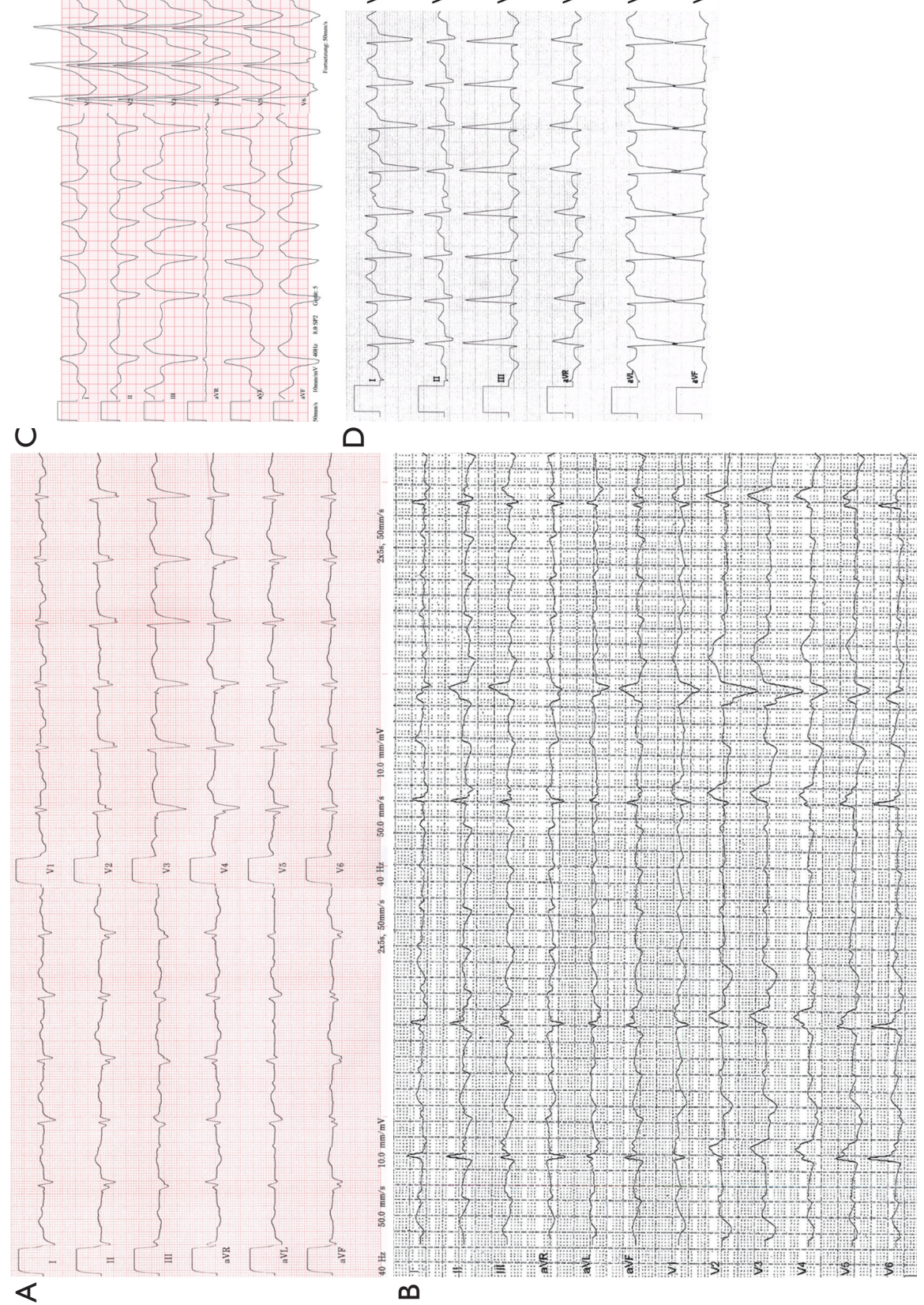

\section{U}
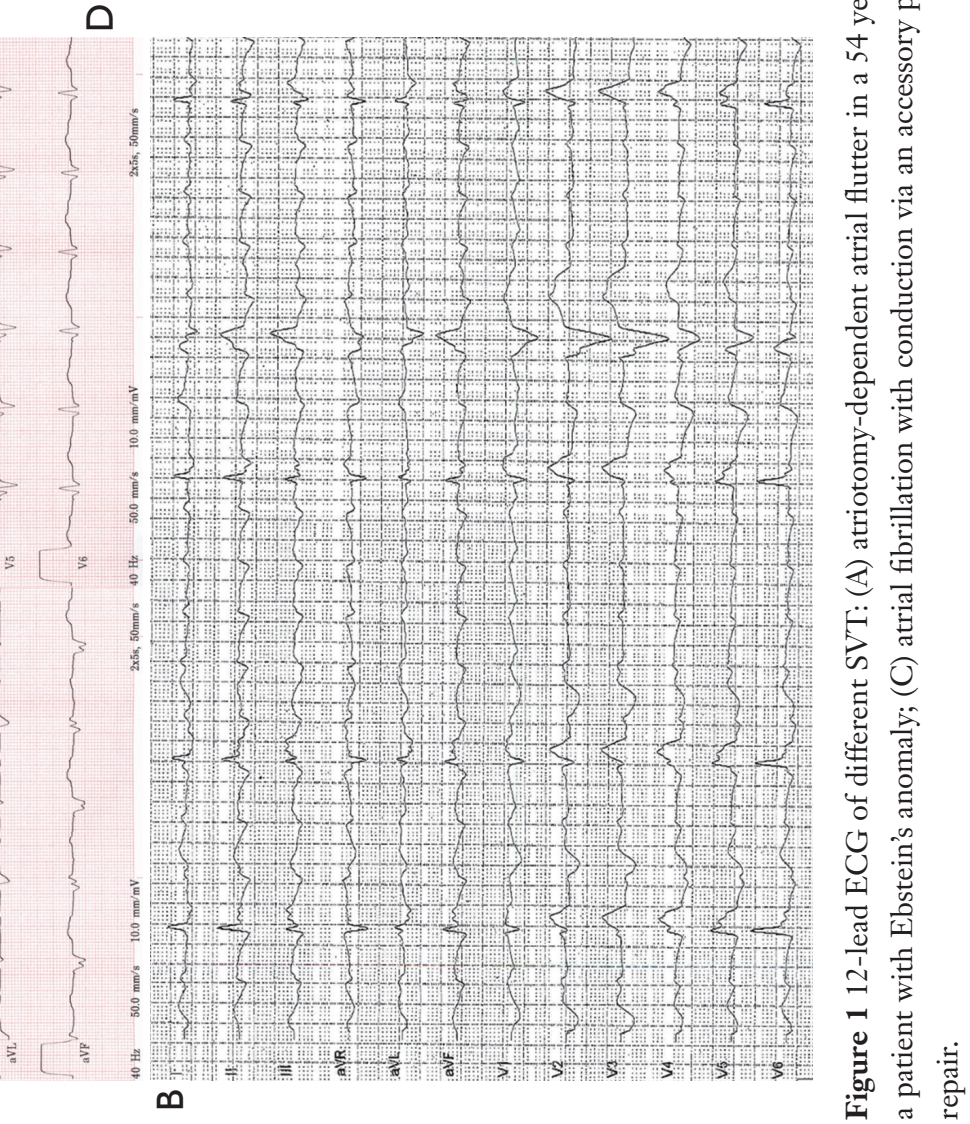
treatment of atrial fibrillation (44) have qualified this perception as being due to patient selection. The DIGITAF trial, an ongoing randomized controlled trial, is currently investigating this issue in patients with heart failure and reduced EF. There are no such trials in patients with CHD. In current ESC guidelines, digoxin has a IIa recommendation for long term rate control.

Rate control in the setting of macroreentrant tachycardias can be difficult to achieve, as atrial activation is organized and often sufficiently slow in the diseased atrial myocardium to allow rapid $\mathrm{AV}$-nodal conduction in these young patients with uncompromised AV-nodes. Particular caution is required in patients with systemic right ventricle as they can rapidly deteriorate hemodynamically, and are at increased risk of heart failure and sudden death due to ventricular arrhythmias. Exclusion of left atrial appendage thrombus is essential prior to elective cardioversion, catheter ablation or antiarrhythmic drug initiation. Anticoagulation has to be established for 4 weeks minimum and long-term in patients perceived at increased stroke risk (see anticoagulation section). If catheter ablation is not possible/available or not desired by the patient, antiarrhythmic drugs can be initiated after electrical cardioversion to prevent arrhythmia recurrences. Choice of antiarrhythmic drug is determined by underlying heart disease. Class Ic drugs can be used in patients with simple CHD without ventricular incisions/ patch material, no relevant ventricular hypertrophy, preserved ventricular function and no severe coronary artery disease. This prerequisite is e.g., present in ASD patients. For all other patients, class III antiarrhythmic drugs, namely amiodarone, are the preferred option.

Antiarrhythmic drugs deliver their effects by slowing conduction in the atrial myocardium and increasing refractoriness. Reentry may subsequently terminate and reinduction can be prevented. However, when using class Ic drugs, atrial activation may be slowed, ultimately allowing 1:1 conduction via the $\mathrm{AV}$-node with rapid heart rates at rest but even more so during exercise. Concomitant AVnode slowing with a beta-blocker is therefore of upmost importance. Flecainide and propafenone are both available for treatment of atrial arrhythmias. While flecainide has no beta-blocking effect, this is variable for propafenone (it is higher in cytochrome PD6-deficient patients) (41). Combination of an $\mathrm{AV}$-node slowing drug with flecainide is therefore generally recommended. Other differences between these two commonly used class Ic drugs include need for dose adjustment in patients with renal dysfunction in patients taking flecainide and interactions with warfarin (increase in INR levels) and digoxin (dose reduction of digoxin required). An advantage of flecainide is the possibility of trough level measurements to evaluate adequate dosing in case of clinical inefficacy. This is not possible for propafenone.

Pre-existing sinus node disease may be unmasked or worsened by antiarrhythmic drugs, particularly class Ic drugs. ECG monitoring should be established until arrhythmia terminates to document any associated bradycardia that requires urgent intervention.

Atrial fibrillation is typically cardioverted electrically after left atrial appendage thrombus exclusion. Antiarrhythmic drugs may terminate arrhythmia or convert atrial fibrillation to atrial flutter. Choice of drug and precautions follow the same rules as for macro-reentrant atrial arrhythmias.

Ventricular arrhythmias require immediate cardioversion or defibrillation, ideally after 12-lead ECG documentation. Catheter ablation may prevent recurrence in monomorphic $\mathrm{VT}$, and an implantable cardioverter defibrillator (ICD) is indicated for secondary prevention.

\section{Pharmacologic prevention of arrbythmia recurrence}

Overall, long-term freedom from arrhythmia with antiarrhythmic drugs is low and side effects limit their long-term use (32). Catheter ablation is therefore first line treatment for patients with AVNRT, AVRT, atrial arrhythmias, and monomorphic VT. Nevertheless, antiarrhythmic drugs are frequently required, particularly if catheter ablation is not successful or not desired by the patient.

Recurrence of paroxysmal SVT including AVNRT and AVRT can be prevented by AV-nodal blocking drugs, predominantly beta-blockers. Class Ic and III drugs are also effective, the same limitations apply as for acute antiarrhythmic drug initiation, namely pro-arrhythmic effects and bradycardia. Rate control in organized atrial arrhythmias can be achieved with beta-blockers, but arrhythmia prevention will typically require initiation antiarrhythmic drugs. Although very effective, side effects are well documented for amiodarone (43). Sotalol can be an alternative despite its inherent risk for proarrhythmia and bradycardia (45).

In patients with symptomatic atrial fibrillation and preserved LV function and no ventricular incision/patch material/hypertrophy, class Ic antiarrhythmic drugs can be prescribed (43). They have to be combined with AV-nodal blocking agents, typically beta-blockers, to control heart 
rate, particularly if atrial flutter develops.

In patients with VT and ICD, concomitant catheter ablation and/or antiarrhythmic drugs may support freedom from arrhythmia recurrence. Amiodarone is most commonly used, but its use is limited by severe side effects. Sotalol may be an alternative. Higher doses ( $\geq 240 \mathrm{mg} / \mathrm{d}$ ) are required to achieve class III effects and QT prolongation and Torsade de pointes are feared complications. Care must be taken in patients with renal dysfunction and dose reduction is recommended in kidney disease (43).

\section{Anticoagulation}

Stroke prevention is of upmost importance in patients with atrial fibrillation and atrial macro-reentrant tachycardias, particularly since stroke risk is already increased in ACHD (46). Routine anticoagulation is generally recommended in patients with atrial fibrillation and moderate or complex CHD. In addition, guidelines recommend oral anticoagulation (OAK) in patients with atrial fibrillation and cyanotic defects, Fontan palliation, and systemic right ventricle $(1,32,47)$. In adults with non-valvular simple forms of $\mathrm{CHD}$ and $\mathrm{AF}$, it appears reasonable to rely on established risk scores. A retrospective study of 229 ACHD patients evaluated $\mathrm{CHA}_{2} \mathrm{DS}_{2}$-VASc score and HAS-BLED score in patients with atrial arrhythmias (48). A score of $\geq 2$ predicted thromboembolic and bleeding risk, respectively, and can therefore be adopted for ACHD.

In the past, vitamin $\mathrm{K}$ antagonists were the mainstay for OAK and there are no comparative studies in ACHD to guide the choice of anticoagulant. Several observational studies now provide reassuring safety data on the use of direct oral anticoagulants (DOAK) in ACHD (49-52). The largest is the NOTE registry that included 530 ACHD patients including 150 with previous vitamin $\mathrm{K}$ antagonist use. Thromboembolic event rate was $1.2 \%$ per year and major bleeding event rate was $1.1 \%$ per year, and not different between DOAK and Vitamin K antagonists. Based on these studies, DOAK appear appropriate for anticoagulation of $\mathrm{ACHD}$ and no mechanical valve.

\section{Catheter ablation}

Supraventricular and monomorphic ventricular tachycardia are amenable to catheter ablation with varying success depending on the underlying defect and type of repair. Although success rate is lower compared to patients without complex heart disease, efficacy is higher compared to pharmacological therapy and prevents patients from side effects associated with antiarrhythmic drug therapy. Complex anatomy and vascular abnormalities may prevent or impede access to the chamber of interest, displaced valves and difficulties in allocation of the $\mathrm{AV}$-node in addition to complex arrhythmia mechanisms and poorly defined circuits are among the challenges of catheter ablation in ACHD. Three-dimensional (3-D) mapping systems (Figure 2), sometimes combined with image integration of MRI or CT scan, are used to identify the underlying mechanism during ongoing or induced arrhythmia and long, steerable sheaths and irrigated catheters with or without contact force measurement to ensure appropriate lesion size and depth are prerequisite for successful ablation in thick and fibrous tissue.

To be sure to address the clinically relevant arrhythmia, we prefer to perform the ablation during ongoing tachycardia. Patients presenting with recurrent IART should therefore not be cardioverted unless hemodynamically unstable but urgently referred to an experienced center for catheter ablation. Nevertheless, induction of atrial arrhythmias at the time of EP-study and ablation of all inducible atrial tachycardias is advocated by some centers, but patients may be non-inducible. Right atrial isthmus ablation is reasonable in these cases, as it is frequently involved in IART. In contrast, patients with AVNRT, AVRT, pre-excitation on 12-lead ECG, atrial fibrillation and monomorphic ventricular tachycardia are studied in sinus rhythm and arrhythmia may be induced and addressed during electrophysiology study.

The more complex the underlying heart defect and type of repair, the more difficult is the procedure and the lower the success rate. Overall acute success rate over the spectrum of supraventricular arrhythmias is $80 \%$, but may be much lower in more complex defects or repair. While different mechanisms of IART in patients with ASD can be readily identified and ablated with a high acute success rate, access to the tricuspid valve in patients with atrial switch procedure is extremely difficult resulting in a lower success rate. Catheter ablation of accessory pathways in patients with Ebstein's anomaly is difficult due to the anatomy and presence of multiple pathways. Long, steerable sheaths and irrigated catheters increase procedural success, which is around $80 \%$ (53).

Monomorphic ventricular arrhythmias occur predominantly in patients with tetralogy of Fallot and VSD (2). In both, anatomical isthmuses underlying VT have been identified and described in detail $(9,10,31)$; (Figure 3). Acute success rate is about $75 \%$, and may be as high as $100 \%$ (2). In some patients, retrograde access and ablation in the aorta may be necessary to achieve complete block in the critical isthmus between VSD 


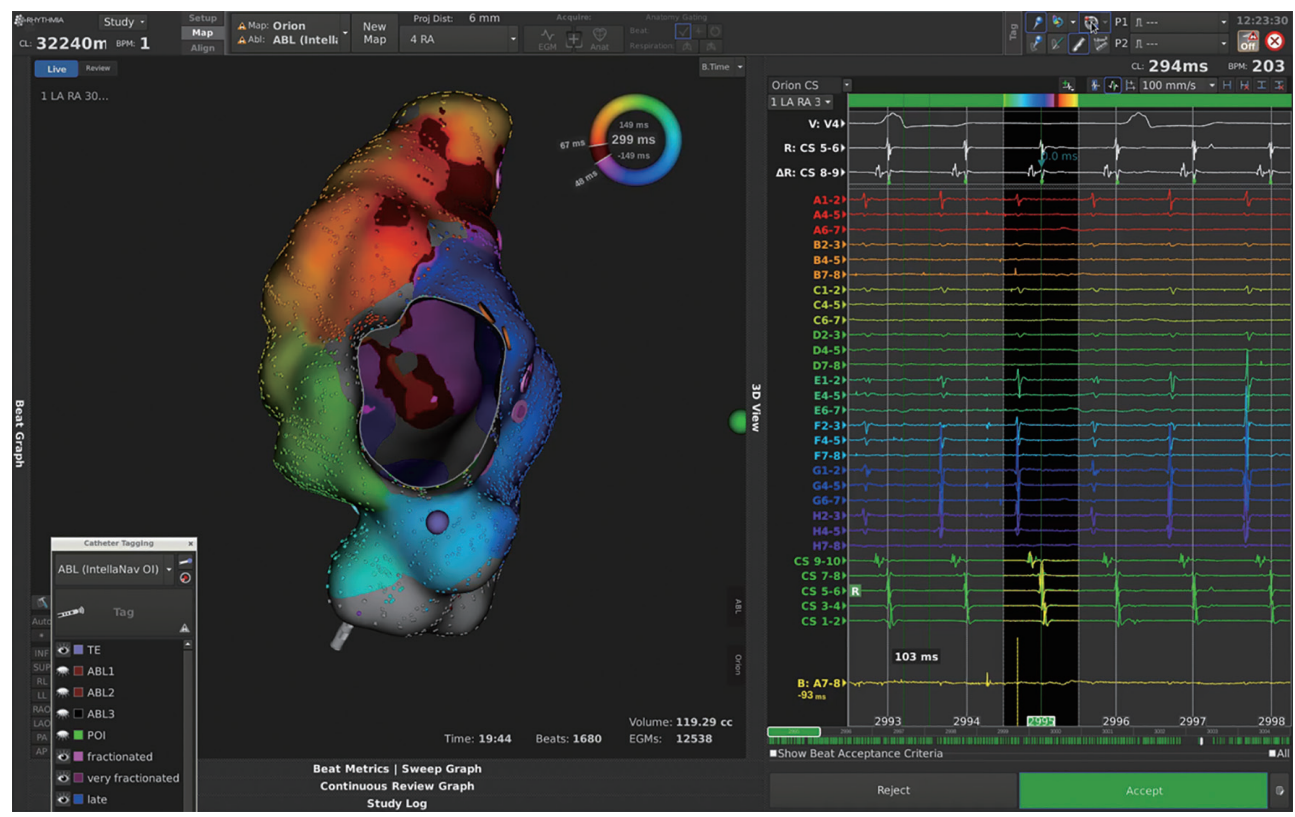

Figure 2 Rhythmia $\mathrm{HDx}^{\mathrm{TM}}$ Map of isthmus dependent right atrial flutter in a patient with Ross-procedure for congenital aortic stenosis. Red is earliest activation and purple/blue is latest activation. LAO view at the tricuspid valve. The activation is counterclockwise around the tricuspid valve. Catheter ablation was successfully performed at the right atrial isthmus between the tricuspid valve and the inferior caval vein.

patch and pulmonary valve (9), as hypertrophy, a homograft or stent material may prevent access to the critical isthmus from the right side. Electrophysiology study and ablation prior to interventional valve replacement and intraoperative surgical treatment of the isthmus is therefore recommended by current guidelines (32). Most patients will be implanted with a defibrillator even after successful catheter ablation to prevent SCD although at least in theory catheter ablation may be curative in this patient cohort (54).

Catheter ablation of SVT and VT can be complicated by AV-block requiring pacemaker implantation and places ACHD at particular risk. In patients with intra-atrial baffles, pacemaker leads may worsen pre-existing baffleobstruction, in patients with single ventricle and patients with Eisenmenger syndrome or lesions with right-to-left shunt, only epicardial pacemaker leads can be implanted with their inherent problems including increased risk during surgical placement in patients with previous heart surgery and lower lead longevity. Adverse outcome of longterm pacing and risk of endocarditis apply to all patients with CHD. Displacement of the AV-node and need for retrograde access in various CHD and arrhythmias increases risk for AV-block in this patient population and requires particular caution.
Atrial fibrillation in ACHD can be treated by catheter ablation as well, but success is limited by underlying substrate. Even in patients with rather simple congenital heart disease like atrial septal defect repair, success rate of pulmonary vein isolation remains low due to the underlying fibrosis. Several small observational series that included between 36 and 84 ACHD with relatively simple CHD in the majority of patients have been published (55-58). Success rates ranged between $32 \%$ and $63 \%$ at one year. Two studies reported success rates at 4 years $(27 \%)$ and 5 years (25\%), respectively. Concomitant use of antiarrhythmic drugs increased success rate to $84 \%$ in one small study with $61 \%$ ASD patients and paroxysmal $\mathrm{AF}$ in $72 \%$. To achieve this success rate, between 1 and 6 procedures were performed, aiming at pulmonary vein isolation as baseline approach and application of additional lesions either during the first procedure or during reablation. The limitations of catheter ablation for $\mathrm{AF}$ in ACHD also apply to the surgical MAZE procedure $(59,60)$. Prophylactic arrhythmia surgery has been advocated to be incorporated into reparative open-heart surgery, but data of efficacy are lacking (32). Incomplete surgical lesions as well as incomplete linear ablation lesions may place patients at risk for organized left atrial arrhythmias which are 

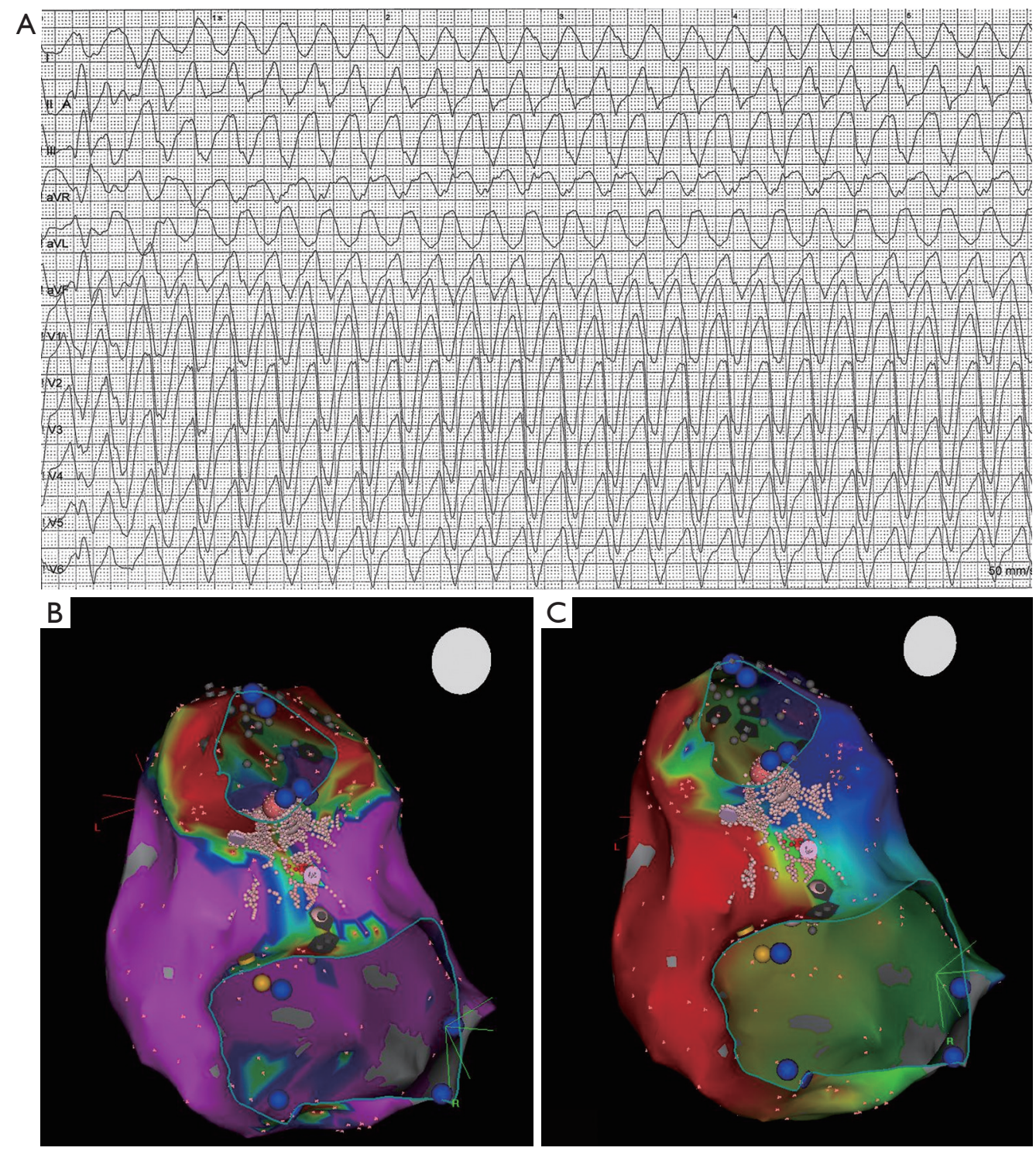

Figure 3 12-lead ECG of induced VT (A), CARTO Voltage Map (B) and (C) activation map during sinus rhythm of a patient with Tetralogy of Fallot and ventricular tachycardia. (A) 12-lead ECG of induced VT in a patient with repaired Tetralogy of Fallot; (B) CARTO Voltage map of the right ventricle from posterior. Red refers to low voltage $(<0.5 \mathrm{mV})$ and purple to normal voltage $(>1.5 \mathrm{mV})$. The blue lines/circles mark the pulmonary valve anulus and the tricuspid valve anulus. Gray refers to scar, here the VSD patch. The yellow ball marks the AV-node where a His signal could be identified. The small pink dots mark where the ablation line was placed to close the gap between the VSD patch and the pulmonary valve, also referred to as Isthmus 4 (31); (C) activation map during sinus rhythm showing slow conduction across isthmus 4. Red is early and purple is late, blue is later than green but earlier than purple.

often much more symptomatic and challenging to achieve appropriate rate control. Nevertheless, before opting for a rate control strategy and prior to committing a young adult with CHD to long-term amiodarone therapy, catheter ablation should be thoughtfully considered. Guidelines recommend consideration of AF ablation in selected patients with simple CHD (32) corresponding to a class IIa recommendation in previous guidelines $(1,61)$.

\section{Conclusions}

Understanding of underlying arrhythmia mechanism in 
specific CHD and surgical repair technique are essential to guide acute and long-term treatment of SVT and VT in ACHD. Catheter ablation is associated with lower acute success rate and higher recurrence rate compared to patients without congenital heart disease but efficacy is still high for many arrhythmias and certainly higher than pharmacological therapy. It should therefore be evaluated for every patient with arrhythmias. Physicians with expertise in treatment of ACHD and clinical electrophysiologists with experience in catheter ablation of ACHD should collaborate to achieve optimal treatment strategy for each individual ACHD patient.

\section{Acknowledgments}

Funding: None.

\section{Footnote}

Provenance and Peer Review: This article was commissioned by the Guest Editors (Yskert von Kodolitsch, Harald Kaemmerer, Koichiro Niwa) for the series "Current Management Aspects in Adult Congenital Heart Disease (ACHD): Part III" published in Cardiovascular Diagnosis and Therapy. The article has undergone external peer review.

Reporting Checklist: The authors have completed the Narrative Review reporting checklist. Available at http:// dx.doi.org/10.21037/cdt-20-634

Conflicts of Interest: All authors have completed the ICMJE uniform disclosure form (available at http:// dx.doi.org/10.21037/cdt-20-634). The series "Current Management Aspects in Adult Congenital Heart Disease (ACHD): Part III" was commissioned by the editorial office without any funding or sponsorship. The authors have no other conflicts of interest to declare.

Ethical Statement: The authors are accountable for all aspects of the work in ensuring that questions related to the accuracy or integrity of any part of the work are appropriately investigated and resolved.

Open Access Statement: This is an Open Access article distributed in accordance with the Creative Commons Attribution-NonCommercial-NoDerivs 4.0 International License (CC BY-NC-ND 4.0), which permits the noncommercial replication and distribution of the article with the strict proviso that no changes or edits are made and the original work is properly cited (including links to both the formal publication through the relevant DOI and the license). See: https://creativecommons.org/licenses/by-nc-nd/4.0/.

\section{References}

1. Khairy P, Van Hare GF, Balaji S, et al. PACES/HRS Expert Consensus Statement on the Recognition and Management of Arrhythmias in Adult Congenital Heart Disease: developed in partnership between the Pediatric and Congenital Electrophysiology Society (PACES) and the Heart Rhythm Society (HRS). Endorsed by the governing bodies of PACES, HRS, the American College of Cardiology (ACC), the American Heart Association (AHA), the European Heart Rhythm Association (EHRA), the Canadian Heart Rhythm Society (CHRS), and the International Society for Adult Congenital Heart Disease (ISACHD). Heart Rhythm 2014;11:e102-65.

2. Brouwer C, Hazekamp MG, Zeppenfeld K. Anatomical substrates and ablation of reentrant atrial and ventricular tachycardias in repaired congenital heart disease. Arrhythm Electrophysiol Rev 2016;5:150-60.

3. d'Udekem Y, Iyengar AJ, Galati JC, et al. Redefining Expectations of Long-Term Survival After the Fontan Procedure. Twenty-Five Years of Follow-Up From the Entire Population of Australia and New Zealand. Circulation 2014;130:S32-8.

4. Lasa JJ, Glatz AC, Daga A, Shah M. Prevalence of arrhythmias late after the Fontan operation. Am J Cardiol 2014;113:1184-8.

5. Li D, Fan Q, Hirata Y, Ono M, An Q. Arrhythmias After Fontan Operation With Intra-atrial Lateral Tunnel Versus Extra-cardiac Conduit: A Systematic Review and Metaanalysis. Pediatr Cardiol 2017;38:873-80.

6. Baysa SJ, Olen M, Kanter RJ. Arrhythmias Following the Mustard and Senning Operations for DextroTransposition of the Great Arteries: Clinical Aspects and Catheter Ablation. Card Electrophysiol Clin. 2017;9:255-71.

7. Khairy P, Clair M, Fernandes SM, et al. Cardiovascular Outcomes After the Arterial Switch Operation for D-transposition of the Great Arteries Circulation 2013;127:331-9.

8. Kapel GFL, Laranjo S, Blom NA, et al. Impact of surgery on presence and dimensions of anatomical isthmuses in tetralogy of Fallot. Heart. 2018;104:1200-7.

9. Kapel GFL, Reichlin T, Wijnmaalen AP, et al. Re-entry 
using anatomically determined isthmuses: a curable ventricular tachycardia in repaired congenital heart disease. Circ Arrhythm Electrophysiol. 2015;8:102-9.

10. Kapel GFL, Sacher F, Dekkers OM, et al. Arrhythmogenic Anatomical Isthmuses Identified by Electroanatomical Mapping Are the Substrate for Ventricular Tachycardia in Repaired Tetralogy of Fallot. Eur Heart J 2017;38:268-76.

11. Bouchardy J, Therrien J, Pilote L, et al. Atrial Arrhythmias in Adults With Congenital Heart Disease Circulation 2009; 120:1679-86.

12. Khairy P, Harris L, Landzberg MJ, et al. Sudden Death and Defibrillators in Transposition of the Great Arteries With Intra-Atrial Baffles: A Multicenter Study. Circ Arrhythm Electrophysiol 2008;1:250-7.

13. de Groot NM, Zeppenfeld K, Wijffels MC, et al. Ablation of Focal Atrial Arrhythmia in Patients With Congenital Heart Defects After Surgery: Role of Circumscribed Areas With Heterogeneous Conduction. Heart Rhythm 2006;3:526-35.

14. Lundqvist CB, Potpara TS, Malmborg H. Supraventricular Arrhythmias in Patients With Adult Congenital Heart Disease. Arrhythm Electrophysiol Rev 2017;6:42-9.

15. Wei W, Zhan X, Xue Y, et al. Features of Accessory Pathways in Adult Ebstein's Anomaly. Europace 2014;16:1619-25.

16. Khositseth A, Danielson GK, Dearani JA, et al. Supraventricular Tachyarrhythmias in Ebstein Anomaly: Management and Outcome. J Thorac Cardiovasc Surg 2004;128:826-33.

17. Takeuchi D, Toyohara K, Shoda M, et al. Electrophysiological Features and Radiofrequency Catheter Ablation of Accessory Pathways Associated With Atrioventricular Discordance. J Cardiovasc Electrophysiol 2020;31:89-99.

18. Gonzalez MD, Sriram CS, Ferre MS. Concordant Location of Accessory Pathways and Tricuspid Valve in AV Discordance. J Cardiovasc Electrophysiol 2020;31:100-2.

19. Epstein MR, Saul JP, Weindling SN, Triedman JK, Walsh EP. Atrioventricular Reciprocating Tachycardia Involving Twin Atrioventricular Nodes in Patients With Complex Congenital Heart Disease. J Cardiovasc Electrophysiol 2001;12:671-9.

20. Liao Z, Chang Y, Ma J, et al. Atrioventricular Node Reentrant Tachycardia in Patients With Congenitally Corrected Transposition of the Great Arteries and Results of Radiofrequency Catheter Ablation. Circ Arrhythm Electrophysiol 2012;5:1143-8.

21. Labombarda F, Hamilton R, Shohoudi A, et al. Increasing
Prevalence of Atrial Fibrillation and Permanent Atrial Arrhythmias in Congenital Heart Disease. J Am Coll Cardiol 2017;70:857-65.

22. Wasmer K, Eckardt L. Management of supraventricular arrhythmias in adults with congenital heart disease. Heart 2016;102:1614-9.

23. Chubb H, Whitaker J, Williams SE, et al. Pathophysiology and manamgement of arrhythmias associated with atrial septal defect and patent foramen ovale. Arrhythm Electrophysiol Rev 2014;3:168-72.

24. Cuypers JA, Opic P, Menting ME, et al. The unnatural history of an atrial septal defect: longitudinal 35 year follow up after surgical closure at young age. Heart 2013;99:1346-52.

25. Kirsh JA, Walsh EP, Triedman JK. Prevalence of and Risk Factors for Atrial Fibrillation and Intra-Atrial Reentrant Tachycardia Among Patients With Congenital Heart Disease. Am J Cardiol 2002;90:338-40.

26. Teuwen CP, Ramdjan TT, Götte M, et al. Time Course of Atrial Fibrillation in Patients With Congenital Heart Defects. Circ Arrhythm Electrophysiol 2015;8:1065-72.

27. Roca-Luque I, Rivas-Gándara N, Dos Subirà L, et al. Long-Term Follow-Up After Ablation of Intra-Atrial ReEntrant Tachycardia in Patients With Congenital Heart Disease: Types and Predictors of Recurrence. JACC Clin Electrophysiol 2018;4:771-80.

28. Tutarel O. Acquired Heart Conditions in Adults With Congenital Heart Disease: A Growing Problem. Heart 2014;100:1317-21.

29. Ebrahim MA, Escudero CA, Kantoch MJ, et al. Insights on Atrial Fibrillation in Congenital Heart Disease. Can J Cardiol 2018;34:1531-3.

30. Upadhyay S, Marie Valente A, Triedman JK, et al. Catheter ablation for atrioventricular nodal reentrant tachycardia in patients with congenital heart disease. Heart Rhythm 2016;13:1228-37.

31. Zeppenfeld K, Schalij MJ, Bartelings MM et al. Catheter Ablation of Ventricular Tachycardia After Repair of Congenital Heart Disease: Electroanatomic Identification of the Critical Right Ventricular Isthmus Circulation 2007;116:2241-52.

32. Hernández-Madrid A, Paul T, Abrams D, et al. Arrhythmias in congenital heart disease: a position paper of the European Heart Rhythm Association (EHRA), Association for European Paediatric and Congenital Cardiology (AEPC), and the European Society of Cardiology (ESC) Working Group on Grown-up Congenital heart disease, endorsed by HRS, PACES, 
APHRS, and SOLAECE. Europace 2018;20:1719-53.

33. Yang H, Kuijpers JM, de Groot JR, et al. Impact of atrial arrhythmias on outcome in adults with congenital heart disease. Int J Cardiol 2017;248:152-4.

34. Mandalenakis Z, Rosengren A, Lappas G, et al. Atrial Fibrillation Burden in Young Patients With Congenital Heart Disease. Circulation 2018;137:928-37.

35. Mandalenakis Z, Rosengren A, Lappas G, et al. Ischemic Stroke in Children and Young Adults With Congenital Heart Disease. J Am Heart Assoc 2016;5:e003071.

36. Saha P, Potiny P, Rigdon J, et al. Substantial Cardiovascular Morbidity in Adults With Lower-Complexity Congenital Heart Disease. Circulation 2019;139:1889-99.

37. Lanz J, Brophy JM, Therrien J, Kaouache M, Guo L, Marelli AJ. Stroke in Adults With Congenital Heart Disease: Incidence, Cumulative Risk, and Predictors. Circulation 2015;132:2385-94.

38. Khairy P. Sudden Cardiac Death in Transposition of the Great Arteries With a Mustard or Senning Baffle: The Myocardial Ischemia Hypothesis. Curr Opin Cardiol 2017;32:101-7.

39. Chaix MA, Chergui M, Leduc C, Khairy P. Sudden death in transposition of the great arteries with atrial switch surgery: Autopsy evidence of acute myocardial ischemia despite normal coronary arteries. Int J Cardiol 2019;288:65-7.

40. Lui GK, Fernandes S, McElhinney DB. Management of Cardiovascular Risk Factors in Adults With Congenital Heart Disease. J Am Heart Assoc 2014;3:e001076.

41. Contractor T, Levin V, Mandapati R. Drug therapy in adult congenital heart disease. Card Electrophysiol Clin 2017;9:295-309.

42. Baumgartner H, De Backer J, Babu-Narayan SV, et al. 2020 ESC Guidelines for the management of adult congenital heart disease. Eur Heart J 2021;42:563-645.

43. Dan GA, Martinez-Rubio A, Agewall S, et al. Antiarrhythmic Drugs-Clinical Use and Clinical Decision Making: A Consensus Document From the European Heart Rhythm Association (EHRA) and European Society of Cardiology (ESC) Working Group on Cardiovascular Pharmacology, Endorsed by the Heart Rhythm Society (HRS), Asia-Pacific Heart Rhythm Society (APHRS) and International Society of Cardiovascular Pharmacotherapy (ISCP). Europace 2018;20:731-2.

44. Hindricks G, Potpara T, Dagres N, et al. 2020 ESC Guidelines for the diagnosis and management of atrial fibrillation developed in collaboration with the European Association for Cardio-Thoracic Surgery (EACTS). Eur
Heart J 2021;42:373-498.

45. Koyak Z, Kroon B, de Groot JR, et al. Efficacy of Antiarrhythmic Drugs in Adults With Congenital Heart Disease and Supraventricular Tachycardias. Am J Cardiol 2013;112:1461-7.

46. Moore BM, Cordina RL, McGuire MA, Celermajer DS. Efficacy and Adverse Effects of Sotalol in Adults With Congenital Heart Disease. Int J Cardiol 2019;274:74-9.

47. Khairy P, Aboulhosn J, Broberg CS, et al. Thromboprophylaxis for Atrial Arrhythmias in Congenital Heart Disease: A Multicenter Study. Int J Cardiol 2016;223:729-35.

48. Baumgartner H, Bonhoeffer P, De Groot NM, et al. ESC Guidelines for the Management of Grown-Up Congenital Heart Disease (New Version 2010). Eur Heart J. 2010;31:2915-57.

49. Heidendael JF, Bokma JP, de Groot JR, Koolbergen DR, Mulder BJM, Bouma BJ. Weighing the Risks: Thrombotic and Bleeding Events in Adults With Atrial Arrhythmias and Congenital Heart Disease. Int J Cardiol 2015;186:315-20.

50. Yang H, Bouma BJ, Dimopoulos K, et al. Nonvitamin K Antagonist Oral Anticoagulants (NOACs) for Thromboembolic Prevention, Are They Safe in Congenital Heart Disease? Results of a Worldwide Study. Int J Cardiol 2020;299:123-30.

51. Pujol C, Niesert AC, Engelhardt A, et al. Usefulness of Direct Oral Anticoagulants in Adult Congenital Heart Disease. Am J Cardiol 2016;117:450-5.

52. Georgekutty J, Kazerouninia A, Wang YF, et al. Novel Oral Anticoagulant Use in Adult Fontan Patients: A Single Center Experience. Congenit Heart Dis 2018;13:541-7.

53. Cheng K, Harrogate S, Orchard E. The Use of Novel Oral Anticoagulants in Adult Congenital Heart Disease: A Single Center Experience. Am J Cardiol 2016;117:312-3.

54. Probst J, Diller GP, Reinecke H, et al. Prevention of sudden cardiac death in patients with Tetralogy of Fallot: Risk assessment and long term outcome. Int J Cardiol 2018;269:91-6.

55. Chetaille P, Walsh EP, Triedman JK. Outcomes of Radiofrequency Catheter Ablation of Atrioventricular Reciprocating Tachycardia in Patients With Congenital Heart Disease. Heart Rhythm 2004;1:168-73.

56. Guarguagli S, Kempny A, Cazzoli I, et al. Efficacy of Catheter Ablation for Atrial Fibrillation in Patients With Congenital Heart Disease. Europace 2019;21:1334-44.

57. Sohns C, Nürnberg JH, Hebe J, et al. Catheter Ablation for Atrial Fibrillation in Adults With Congenital Heart 
Disease: Lessons Learned From More Than 10 Years

Following a Sequential Ablation Approach. JACC Clin Electrophysiol 2018;4:733-43.

58. Liang JJ, Frankel DS, Parikh V, et al. Safety and Outcomes of Catheter Ablation for Atrial Fibrillation in Adults With Congenital Heart Disease: A Multicenter Registry Study. Heart Rhythm 2019;16:846-52.

59. Philip F, Muhammad KI, Agarwal S, Natale A, Krasuski RA. Pulmonary Vein Isolation for the Treatment of Drug-

Cite this article as: Wasmer K, Eckardt L, Baumgartner H, Köbe J. Therapy of supraventricular and ventricular arrhythmias in adults with congenital heart disease-narrative review. Cardiovasc Diagn Ther 2021;11(2):550-562. doi: 10.21037/cdt-20-634
Refractory Atrial Fibrillation in Adults With Congenital Heart Disease. Congenit Heart Dis 2012;7:392-9.

60. Gonzalez Corcia MC, Walsh EP, Emani S. Longterm Results of Atrial Maze Surgery in Patients With Congenital Heart Disease. Europace 2019;21:1345-52.

61. Mavroudis C, Stulak JM, Ad N, et al. Prophylactic Atrial Arrhythmia Surgical Procedures With Congenital Heart Operations: Review and Recommendations Ann Thorac Surg 2015;99:352-9. 